\title{
Impurity-Semiconductor Band Hybridization Effects on the Critical Temperature of Diluted Magnetic Semiconductors.
}

\author{
M. J. Calderón ${ }^{1,3}$, G. Gómez-Santos², and L. Brey ${ }^{3}$. \\ ${ }^{1}$ Cavendish Laboratory, Cambridge University, Madingley Road, Cambridge CBз оHE, UK. \\ Condensada and Instituto Nicolás Cabrera, Universidad Autónoma de Madrid, 28049 Madrid, Spain. \\ and \\ ${ }^{3}$ Instituto de Ciencia de Materiales de Madrid (CSIC), Cantoblanco, 28049, Madrid, Spain.
}

\begin{abstract}
aty
We have studied the critical temperature of Diluted Magnetic Semiconductors by means of Monte Carlo simulations and Coherent-Potential-Approximation (CPA) calculations. In our model for this system, the magnetic ions couple with the carriers through an antiferromagnetic exchange interaction, $J$, and an electrostatic interaction $W$. The effective impurity potential $J-W$ controls the hybridization between the magnetic impurities and the hole charge on the dopants. We find that the critical temperature depends substantially on the hole charge on the magnetic impurities. The CPA critical temperature is always lower than the obtained in the Monte Carlo simulations, although all trends in the simulation results are reproduced in the CPA calculations. Finally we predict the existence of pockets of phase segregation instability close to the carriers band edges.
\end{abstract}

PACS numbers: 75.50.Pp, 75.10Lp

\section{INTRODUCTION.}

It has been recently discovered that some $\mathrm{Mn}$ doped semiconductors, such as $\mathrm{Ga}_{1-x} \mathrm{Mn}_{x} \mathrm{As}$, undergo a ferromagnetic-paramagnetic transition at temperatures near 100K [1, 2]. Motivated by this high Curie temperature, there have been further experimental studies in III-V magnetic semiconductors, and ferro-paramagnetic transition temperatures as high as $1000 \mathrm{~K}$ have been obtained in $(\mathrm{Ga}, \mathrm{Mn}) \mathrm{N}$ [3]. The optimal Mn concentration is near $5 \%$ in these materials, making magnetic ions to be rather diluted in the host semiconductor.

The existence of ferromagnetic semiconductors at room temperature opens the possibility of combining the spatial modulations of both carrier and spin density. Therefore, diluted magnetic semiconductors (DMS) have attracted much attention for their potential use in spintronic devices. Some spintronic devices have been already implemented [ $\mid 1$, and different heterostructures in order to optimize and control the critical temperature have been proposed [5, 6, 7, 8].

In this work we study magnetic properties III-V magnetic semiconductors, in particular of GaMnAs. The high critical temperature DMS's have a high concentration of randomly located manganese ions. From electron paramagnetic resonance and optical experiments [9, 10], it is known that the Mn ions in these semiconductors have a $S=5 / 2$ local moment. The Mn ions go in substitutionally at the cation sites, Ga, and contribute holes to the semiconductor valence band. Although the local moment of the magnetic ions in GaMnAs indicates that each Mn should contribute with a hole, it is experimentally observed that the density of itinerant holes, $p$, is a small fraction $(\sim 10 \%)$ of the Mn concentration [11]. The reduction in the carrier density is probably due to the presence of antisite defects which, acting as deep donors partially compensating the Mn acceptors, reduce the hole density [12]. A large number of antisite defects is expected to be present in these systems because, in order to minimize the mobility of the $\mathrm{Mn}$ ions and the existence of clusters, the semiconductors are fabricated using molecular beam epitaxy growing techniques at low temperatures, for which a large number of these defects is known to occur 12, 13.

It is widely accepted that the ferromagnetism in III-V semiconductors is induced by the motion of the electrical carriers in the system. However, there is no agreement on the precise role of the carriers. They can be either itinerant, with a clear character of the host semiconductor valence band states 14, 15, 16, 17], or rather they can be mostly located on Mn impurities, with a clear impurity band magnetic, polaron character 18, 19, 20, 21. On the other hand, the carrier induced ferromagnetic interaction between $\mathrm{Mn}^{2+}$ ions in magnetic semiconductors has some resemblance to the one observed in manganese perovskites [22], double perovskites [23], and pyrochlores [24. In these systems, the phase diagram is different from that of conventional itinerant ferromagnets, showing first-order transitions, phase separation [25], and formation of magnetic polarons near the Curie temperature 26, 27.

In order to study these issues, we use a tight-binding model Hamiltonian in which, besides the Hund's coupling, a site energy difference between magnetic and normal ions, $W$, is introduced. Monte Carlo (MC) simulations are then performed for this model, with particular emphasis on the $W$ dependence of the phase diagram. A mean field calculation within the coherent-potentialapproximation (CPA) is then carried out and compared with $\mathrm{MC}$ results. The CPA treatment confirms the robustness of the MC results for the effect of $W$, in addition 
to allowing a simpler exploration of important issues such as the existence of phase separation.

Our main results are the following:

1) The hole charge on the Mn ions, controlled by the impurity potential $W$, has a profound effect on the transition temperature. Upon increasing $W$, the hole charge on Mn decreases, leading to a substantial decrease of the Curie temperature.

2) For much of the model's parameter space, the MC critical temperature is higher than that obtained using the simplest Weiss mean field theory. The MC critical temperature is always higher than the corresponding CPA temperature. Nevertheless, all trends in the MC results, particularly the effect of the impurity potential, are reproduced in the CPA calculation. We believe this to be a robust feature.

3) The CPA calculation shows the existence of small pockets of phase segregation instability close to band edges.

The paper is organized in the following way. In section II we introduce and explain the model. Section III and IV describe the MC and CPA methods, respectively. In Section V we present our results, both MC and CPA, making contact with previous calculations and/or experiments. Section VI summarizes our work.

\section{THE MODEL.}

It is generally accepted $14,15,16,17,18,19,20$ that the DMS's are governed by the following Hamiltonian:

$$
H=H_{h}+H_{\text {ions }}+H_{h-i o n s},
$$

$H_{h}$ and $H_{\text {ions }}$ are the parts of the Hamiltonian describing the holes and the Mn ions, respectively. The term $H_{h-i o n s}$ represents the interaction between the magnetic ions and the carriers.

$H_{h}$ is the sum of the kinetic energy of the carriers, the hole-hole interaction energy, and the interaction of the holes with the disorder potential from randomness in the host semiconductor, in particular from the presence of antisite defects. In the actual magnetic semiconductors, the carrier density is of the order of $10^{20} \mathrm{~cm}^{-3}$, and, for these values, it is justified to neglect the effect of the carrier-carrier interaction. In this work we do no take into account the interaction of carriers with antisite disorder. This contribution is probably important in actual samples, but here we are mainly interested in the effect of the on-site energy difference and thermal fluctuations on the critical temperature. We describe the motion of the holes using a single one-band hole dispersion, instead of the more appropriated six bands envelope function formalism [28]. This one-band model is probably insufficient to give a quantitative prediction for the Curie temperature, but it includes the main features of the hole system we want to study: the effect of thermal fluctuations, Mn-GaAs orbital hybridization, and charge localization effects. With all this, the Hamiltonian for the holes is written as

$$
H_{h}=-t \sum_{<i, j>}\left(C_{i, \sigma}^{+} C_{j, \sigma}+\text { h.c. }\right)
$$

where the sum runs over all first neighbors pairs on a simple cubic lattice, $t$ is the tunneling amplitude, and $C_{i, \sigma}^{+}$creates a hole with spin $\sigma$ at site $i$. This model has a hole bandwidth of $12 t$, and the parameter $t$ has been chosen to give a bandwidth of the order of that of GaAs $(\sim 10 \mathrm{eV})$, implying $t \sim 0.84 \mathrm{eV}$. The band edges of this model correspond to a parabolic dispersion with an effective mass $m^{*}=\hbar^{2} / 2 a^{2} t$, being $a$ the lattice parameter of the simple cubic lattice. The value of $a$ has been chosen to make $a^{3}$ the volume per GaAs unit, i.e. $a=3.56 \AA$. With these parameters, we obtain an effective mass $m^{*}=0.36 m_{0}$.

The term $H_{\text {ions }}$ in Eq.(1) describes the direct antiferromagnetic interactions between the magnetic moments of the Mn ions. Those are much smaller than the interactions with the carrier spins and, therefore, we neglect them.

Finally, the term $H_{h-i o n s}$ represents the coupling between the electrical carriers and the magnetic impurities. There are two contributions to this term: i) An antiferromagnetic exchange interaction between the spins $\mathbf{S}_{I}$ of the $\mathrm{Mn}^{2+}$ ions located at sites $I$ and the spins of the itinerant carriers. This interaction produces a long range ferromagnetic interaction between the $\mathrm{Mn}$ ions. And ii) an interaction between the carriers charge and the potential arising from the Mn dopants:

$$
H_{h-i o n s}=\frac{J}{S} \sum_{I} \mathbf{S}_{I} C_{I, \alpha}^{+} \boldsymbol{\sigma}_{\alpha, \beta} C_{I, \beta}+W \sum_{I} C_{I, \sigma}^{+} C_{I, \sigma} .
$$

Here the sum runs over the positions of the magnetic impurities, and $\boldsymbol{\sigma}$ are the Pauli matrices. The origin of $W$ is Coulombic, coming from the different electronegativity of $\mathrm{Mn}$ and GaAs atoms, and from the screening of the impurities by the electrical carriers. The quantity $J-W$ governs the hybridization of the magnetic impurities with the host bands, and the excess of charge on the impurities with respect the host semiconductor atoms. For instance, in the case of a perfect ferromagnetic ground state, the electrical charge is uniformly distributed for $J=W$. The value of $J$ in our model is connected with the exchange coupling $J_{p d}$ appearing in the continuum model 29. through $J=S J_{p d} / 2 a^{3}$. The value of $J_{p d}$ is typically $0.05-0.1 \mathrm{eV} \mathrm{nm^{3 }}$ [1, 2, 30, 31], yielding $J \sim 1.4-$ $2.8 \mathrm{eV}$, which gives a value of $J / t \sim 1.7-3.4$. Local spin density calculations (LSDA) and supercell methods 32] applied to GaMnAs predict a value of $J$ rather larger than the experimental one, partially because the LSDA calculations overestimate exchange coupling strengths. Lacking reliable experimental information on the value of $W$, we consider it as a parameter with values between 0 and $J$. Summarizing the model: we restrict ourselves 
to the terms $H_{h}$ and $H_{h-i o n s}$, given by Eq. 2 and Eq. 3 , respectively.

\section{DESCRIPTION OF THE MONTE CARLO ALGORITHM.}

We have performed classical MC simulations on the Mn core spin angles in order to calculate the temperature, $T$, dependent magnetic phase diagram. The simulations are done in a $N \times N \times N$ cubic lattice with periodic boundary conditions, and $N_{M n}=x N^{3}$ randomly located magnetic ions. The number of carriers is taken to be $N_{h}=p N^{3}$.

The simulations are done as follows. First, we produce a realization of the disorder: the positions of the Mn ions. For this realization, we compute the expectation value of the hole spin orientation at every place,

$$
\mathbf{n}_{i}=<C_{i, \alpha}^{+} \boldsymbol{\sigma}_{\alpha, \beta} C_{i, \beta}>.
$$

At a given temperature, the Mn spins fluctuate thermically on a fictitious magnetic field [33] proportional to the carriers spin density,

$$
\mathbf{B}_{f}(I)=\frac{J}{S} \mathbf{n}_{I}
$$

We perform MC simulations on the Mn spin angles until the system is equilibrated, when we choose a particular core spin configuration, and recalculate the hole spin density. This cycle is repeated until convergence in physical quantities is attained. Calculating the hole spin density requires the diagonalization of the Hamiltonian Eq.(11) at each step of the cycle. $\mathbf{n}_{i}$ is evaluated by filling up the $N_{h}$ lowest energy levels of the Hamiltonian. This can be done because the Fermi temperature is much higher than any other temperature in the system. For instance, for $x=10 \%, p=1 \%$, and $J=W=3 t$, the Fermi energy is near $0.5 t$, while the Curie temperature is $\sim 0.015 t$.

The CPU diagonalization cost grows as $N^{6}$, severely limiting the system's sizes under study. By diagonalizing the Hamiltonian in real space, it is not possible to analyze systems bigger than $N=6$ [34, 35]. In order to study bigger systems, it is better to use an adapted basis rather than the atomic localized basis. In the simulation, we use the basis obtained by diagonalizing the hole Hamiltonian for a perfect ferromagnetic alignment of the Mn ion spins. For the updating of the electron spin density at each cycle of the simulation, it is not necessary to include all the eigenvectors of the extended basis. We have found enough to work with a reduced basis formed by the $N_{c}$ lowest energy eigenstates of this basis for each carrier spin orientation. The value of $N_{c}$ depends on the particular values of the parameters. In general, we find that the size of the required basis increases with the value of $J-W$, i.e. it increases with the degree of localization of the hole charge on the Mn ions. Typically we can obtain good convergence with relatively small values of $N_{c}$, most of the results presented in this paper have been obtained with $N_{c}=57$. Note that, in the extended basis we use, the square of the wave functions is not spatially uniform, as it would happen in a Bloch or plane wave basis, but it is modulated by the spatial fluctuations of the potential felt by the carriers. Using this extended basis and an appropriate cutoff $N_{c}$, we can perform MC simulations in systems with sizes up to $\mathrm{N}=16$.

In the simulations, we calculate the thermal averages of different quantities: the absolute value of the Mn ions spin polarization,

$$
M=\frac{1}{S N_{M n}}\left\langle\left|\sum_{I} \mathbf{S}_{I}\right|\right\rangle,
$$

the absolute value of the hole's spin polarization,

$$
m=\frac{1}{N_{h}}\left\langle\left|\sum_{i} \mathbf{n}_{i}\right|\right\rangle,
$$

and the average value of the hole charge on the Mn ions,

$$
Q_{M n}=\frac{1}{N_{M n}}\left\langle\sum_{I} \rho_{I}\right\rangle,
$$

being $\rho_{i}=<\sum_{\sigma} C_{i, \sigma}^{+} C_{i, \sigma}>$ the electrical hole charge on site $i$. In the previous expressions, the index $I$ runs over the Mn positions and $i$ over all lattices sites.

\section{CPA METHOD.}

We have performed mean field calculations for the present model within the framework of the CoherentPotential-Approximation (CPA) 36, 37, 38]. In recent times, this formalism has come to be known as the Dynamical-Mean-Field-Approximation (DMFA) [39], and we use both terms (CPA and DMFA) as synonymous expressions. Given the possible confusion from the use of different flavors and names under the common umbrella of mean field calculations, we devote this section to briefly describe our CPA formalism, pointing out the origin and nature of the approximations and, where appropriate, making contact with other approaches.

Tracing out the fermions at constant chemical potential and temperature, we end up with a probability distribution for the core spins given by the following Boltzmann weight:

$$
\mathcal{P}\left(\left\{\mathbf{S}_{i}\right\}\right) \propto \exp \left[-\beta \Omega\left(\left\{\mathbf{S}_{i}\right\}\right)\right]
$$

where $\Omega\left(\left\{\mathbf{S}_{i}\right\}\right)$ is the carriers grand canonical potential for a given core-spin configuration. The exact distribution $\mathcal{P}\left(\left\{\mathbf{S}_{i}\right\}\right)$ minimizes the following thermodynamical potential, considered as a functional over spin distributions:

$$
\mathcal{F}[\mathcal{P}]=\left\langle\Omega\left(\left\{\mathbf{S}_{i}\right\}\right)\right\rangle+T\langle\log (\mathcal{P})\rangle
$$

where averages are taken over core-spin distributions. This formulation offers a convenient starting point for approximate variational approaches. 
The common basis of all mean field calculations is the minimization of Eq. 10 with a factorization ansatz for the spin distribution:

$$
\mathcal{P}\left(\left\{\mathbf{S}_{i}\right\}\right)=\prod_{i} p\left(\mathbf{S}_{i}\right)
$$

From the critical phenomena point of view, this factorization legitimizes the term mean field, implying that all criticality (if any) will be classical. Carrying out the minimization of Eq. 10 with the only simplification of the factorization could be termed the absolute mean field approximation. Unfortunately, this procedure leaves us with the task of calculating the grand canonical potential for fermions (i.e. the density of states) in a sitedisordered background: a formidable problem requiring numerical treatments (see, for instance, Ref. 40] for such a treatment in the Double-Exchange model).

To cope with the previous difficulty, additional approximations are needed to obtain the average density of states for the disordered problem. We will describe two of them, namely, the Virtual-Crystal-Approximation and CPA treatment (see Ref. 38 for a review on disordered systems).

i) Virtual Crystal Approximation (VCA). The average density of states for the disordered system is replaced by that of the average Hamiltonian. That implies a translationally invariant system with site energies given by the following average (a $2 \times 2$ matrix in spin space): $\varepsilon_{\alpha, \beta}=x\left(W+J \frac{\langle\mathbf{S}\rangle}{S} \cdot \boldsymbol{\sigma}_{\alpha, \beta}\right)$. The minimization of $\mathcal{F}$ is now straightforward, leading to the following well-known expression for the transition temperature:

$$
T_{c}^{V C A}=\frac{2}{3} x J^{2} n_{\sigma}(\mu)
$$

where $n_{\sigma}(\mu)$ is the density of states per site and spin at the Fermi level (degenerate fermions assumed). This scheme is often referred to as the Weiss mean field approach, and we will consider both expressions (Weiss mean field and VCA) as synonymous terms.

ii) Coherent Potential Approximation (CPA). The CPA is a time-honored approach for the site-disordered "alloy" problem [36, 37]. It is known to produce good results, particularly in three dimensions [38]. The averaged Green's function is obtained from a translationallyinvariant, effective medium, characterized by a (sitediagonal and energy dependent) self-energy $\Sigma_{\alpha, \beta}(z)$ in the following manner:

$$
\langle G(z)\rangle \simeq G^{C P A}=\left(z-H_{C P A}\right)^{-1}
$$

where (Dirac notation used to emphasize the one-body character of the problem)

$$
H_{C P A}=-t \sum_{(i, j)}|i, \sigma\rangle\left\langle j, \sigma\left|+\sum_{i}\right| i, \alpha\right\rangle \Sigma_{\alpha, \beta}(z)\langle i, \beta|
$$

with sum over spin indices implied. Given a particular site-disorder distribution, the self-energy is obtained with a minimum scattering requirement: the substitution of an effective site by a real one should produce zero (local) scattering on the average 41. That is:

$$
\left\langle\left(\hat{\varepsilon}_{i}-\hat{\Sigma}\right)\left[\hat{1}-\hat{G}_{i i}^{C P A}\left(\hat{\varepsilon}_{i}-\hat{\Sigma}\right)\right]^{-1}\right\rangle=0
$$

where hats mean $2 \times 2$ matrices in spin space, and the random quantities $\hat{\varepsilon_{i}}$ are given by:

$$
\varepsilon_{i(\alpha, \beta)}=\left\{\begin{array}{clrl}
0, & & \text { probability } & =(1-x) \\
W+J \frac{\mathbf{S}_{i}}{S} \cdot \boldsymbol{\sigma}_{\alpha, \beta}, & \text { probability } & =x p\left(\mathbf{S}_{i}\right)
\end{array}\right.
$$

As usual, the minimization of the thermodynamic potential can be cast in the form of a self-consistency equation for the probability distribution:

$$
p\left(\mathbf{S}_{i}\right) \propto \exp \left[-\beta \delta \Omega\left(\mathbf{S}_{i}\right)\right]
$$

where $\delta \Omega\left(\mathbf{S}_{i}\right)$ can be interpreted as the total change in the fermion's grand canonical potential for embedding an impurity site in the effective medium:

$$
\delta \Omega\left(\mathbf{S}_{i}\right)=\int d \omega f(\omega) \frac{1}{\pi} \Im \log \operatorname{det} \hat{M}\left(\mathbf{S}_{i}\right)
$$

$f(\omega)$ is the Fermi-Dirac factor, $\Im$ means imaginary part, and $\hat{M}\left(\mathbf{S}_{i}\right)$ is given by:

$$
\hat{M}\left(\mathbf{S}_{i}\right)=\hat{1}-\hat{G}_{i i}^{C P A}\left(\omega^{+}\right)\left[\hat{\varepsilon}\left(\mathbf{S}_{i}\right)-\hat{\Sigma}\left(\omega^{+}\right)\right]
$$

It is through eqs. 17 and 18 that the CPA can be connected with the DMFA (see, for instance, Ref. 44), an approach primarily intended for genuine many-body problems like the Hubbard model 39. It has been recently applied to models of itinerant carriers with ion impurities, believed to be relevant for manganites and doped magnetic semiconductors (see, for instance, Ref. 42, 43, 44, 45]).

Before leaving this section, let us comment on some features of these approaches. It is clear that the VCA is simple and handy, but the approximation in the density of states is too drastic. For instance, it predicts a ferromagnetic transition for any finite density of states. This cannot be true, even at the mean field level: for $W=0$ and small $J$, the problem becomes the standard Ruderman-Kittel-Kasuya-Yosida (RKKY) Hamiltonian, whose absolute mean field treatment shows ferromagnetism only for carrier density (holes or electrons) below a critical value $p_{c}=0.5038$ (sc lattice), due to the oscillatory behavior of the spin-spin coupling. Furthermore, the VCA is not sensitive to the value of $W$, obviously a major limitation for this problem. In contrast, the CPA is free from these shortcomings. For instance, it is able to reproduce the absolute mean field results of the RKKY limit 46. In addition, extensive numerical results available for the exact density of states in the closely related Double-Exchange problem [40], show virtually no differences with the CPA density of states 47. This reinforces our confidence in this method for the present problem. 


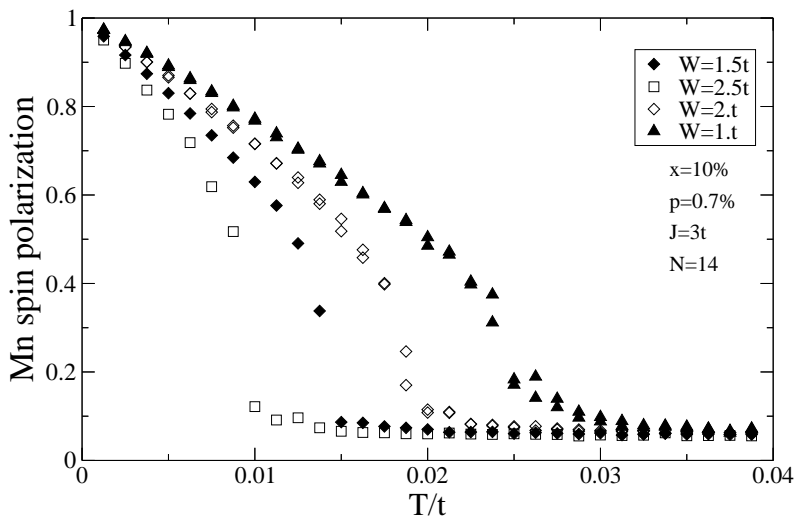

FIG. 1: Mn ions spin polarization as a function of temperature for $J=3 t, x=10 \%, p=0.7 \%, N=14$ and different values of the parameter $W$. Results for different realizations of disorder are plotted.

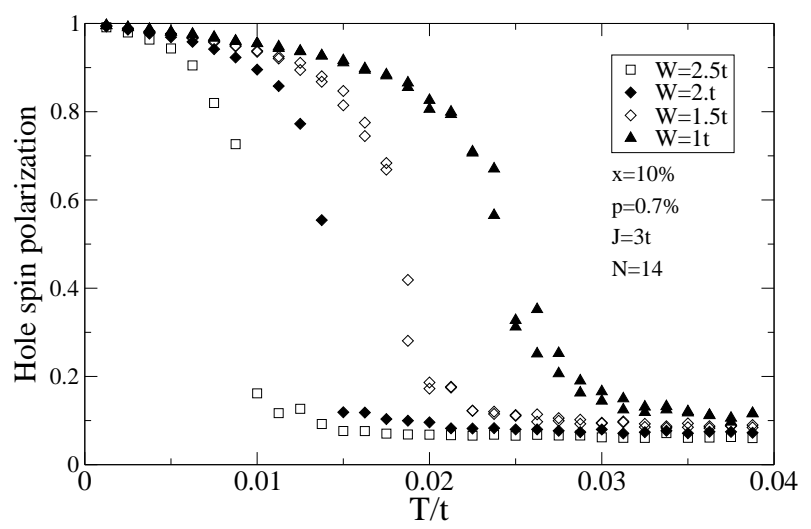

FIG. 2: Holes spin polarization as a function of temperature for $J=3 t, x=10 \%, p=0.7 \%, N=14$ and different values of the parameter $W$. Results for different realizations of disorder are plotted.

\section{RESULTS.}

\section{A. Monte Carlo Results.}

In Fig. 11 we plot the Mn ion's spin polarization, $M$, as a function of $T$, for $J=3 t$, and different values of $W$. The results correspond to a Mn concentration $x=10 \%$, a hole concentration $p=0.7 \%$, and a unit cell size $N=14$. In Fig. 2 we plot the hole's spin polarization for the same set of parameters. Due to the finite size effects (note that there are only $274 \mathrm{Mn}$ ions) the polarizations $M$ and $m$ are different from zero at any temperature, and we define the critical temperature, $T_{c}$, as the point where the second derivate of the polarization with respect to $T$ changes sign. This way of obtaining $T_{c}$ implies uncertainties of around $5 \%$. Within this error margin, the $T_{c}$ 's obtained from the temperature dependence of $m$ and $M$ coincide. This is the expected result as the carrier's spin polarization is due to the Mn polarization.

Comparing Fig. 1 and Fig. 2, we see that the ion spin

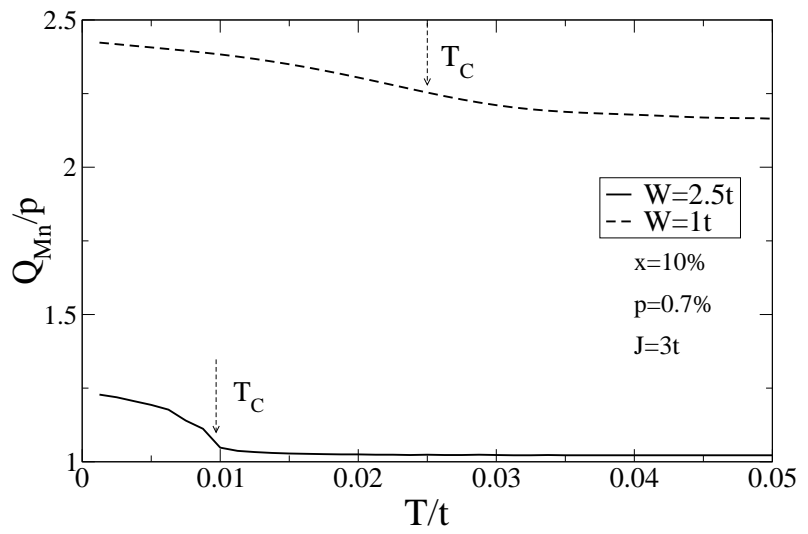

FIG. 3: Average hole charge on Mn ions, as a function of $T$, for two different values of $W$. The results corresponds to the case $J=3 t, x=10 \%, p=0.7 \%$, and $N=14$. The arrows mark the Curie temperature, as obtained from Fig. 1 and Fig. 2.

polarization decreases faster with temperature than that of the holes. This seems to be generally true for the range of hole and Mn densities of interest, and a value of $J=3 t$. This behavior has been already found in Weiss mean field calculations [8]. Different disorder realizations are superimposed in figures 1 and 2 . We find that, for small values of $J-W$, different disorder realizations give practically the same polarization curves, whereas, for small values of $W$, fluctuations related with disorder become more important. This is because the larger $J-W$, the deeper the effective impurity level. This increases the charge localized in the Mn ions, making the fluctuations associated with impurity randomness more important.

The critical temperature decreases when $W$ increases. As we will show, this is related to the amount of hole charge localized on Mn ions. In Fig. 3 we plot the average hole charge on $\mathrm{Mn}$ as a function of $T$, for two different values of $W$. Fig. 3 shows that the charge on the Mn's decreases as the effective impurity potential increases, that $Q_{M n}$ decreases with temperature, and that there is a change in the sign of the second derivate of $Q_{M n}$ with respect to temperature at $T_{c}$.

The delocalization of charge with increasing $T$, and its abrupt change at $T_{c}$, indicate a possible change in transport properties of the system near the ferro-paramagnetic transition: we expect that the electrical resistivity of the system will decrease when $T$ increases. For a value of $W=J$, the effective impurity potential is exactly zero in the perfect ferromagnetic state. Close to this value, the charge on each $\mathrm{Mn}$ ion is practically $p$, independently of $T$. However, for values of $W$ near zero, the charge on the Mn ions is very large, more than three times $p$. Note that, in the case of $W=0$, the critical value of $J$ for binding a hole of the appropriate spin to a $\mathrm{Mn}$ ion is $J_{c}=3.95677 t$. For values of $J>J_{c}$, the holes form a parallel-spin impurity band split from the main general band [45]. In that case, the physics becomes more similar to that of the Double-Exchange materials. In our model, 


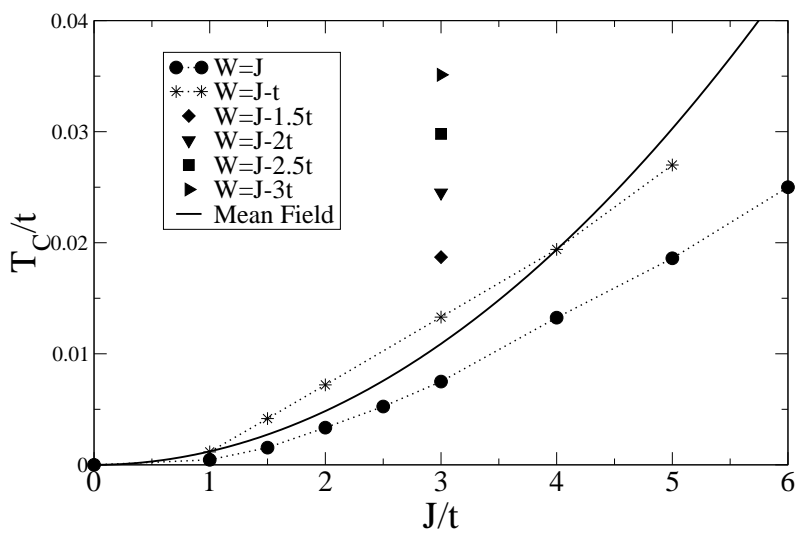

FIG. 4: Critical temperature as a function of $J$ for different values of $W$. The results corresponds to the case $x=10 \%$, $p=0.7 \%$, and $N=14$. The Weiss mean field critical temperature is plotted.

the parameter $W$ allows us to modulate the hybridization between the Mn ions orbitals and the host semiconductor band. In the case of $J=3 t$, we believe that a lower impurity band is not formed for any positive value of $W$.

In Fig. 国we plot the critical temperature obtained from MC simulations as a function of $J$ for different values of $W$, and for concentrations $p=0.7 \%$ and $x=10 \%$. We have also plotted the critical temperature, Eq. 12, obtained in the Weiss mean field theory [14, 15]. The Weiss mean field $T_{c}$ increases quadratically with $J$ and, as explained before, the approximation treats the magnetic impurities in the virtual crystal approximation, leading to a $T_{c}^{V C A}$ independent of $W$. From the MC results, we observe that the MC critical temperatures are smaller than the $T_{c}^{V C A}$ 's only for values of $W$ very close to $J$. This is because, for $W=J$, the effective impurity potential is zero, and the virtual crystal approximation for obtaining the Curie temperature seems to work well. In this case, $W=J$ and small $J$, the thermal fluctuation correction to $T_{c}^{V C A}$ is similar to that occurring in the standard Heisenberg model, $\sim 25 \%$. This becomes clear in Fig. 5, where we plot $M(T)$ as obtained in MC simulation and compare it with the predictions of Weiss mean field. Both curves are very similar at low $T$, and only near the Curie temperature do they separate, due to thermal fluctuation effects. For small values of $W$, the MC critical temperatures are larger than the $T_{c}^{V C A}$ 's. This is due to the charge located on the $\mathrm{Mn}$ ions which increases the effective magnetic field felt by the Mn spins.

If the effective impurity potential $J-W$ increases, holes would eventually form a localized impurity band, leading to a decrease of $T_{c}$. As commented above, we do not get close enough to this limit, whose MC description would require a large basis beyond our present computational capabilities.

We have also studied the dependence of the critical temperature on the Mn concentration, $x$. This is shown in Fig. 6, where we plot $T_{c}$ as a function of $x$ for $J=3 t$,

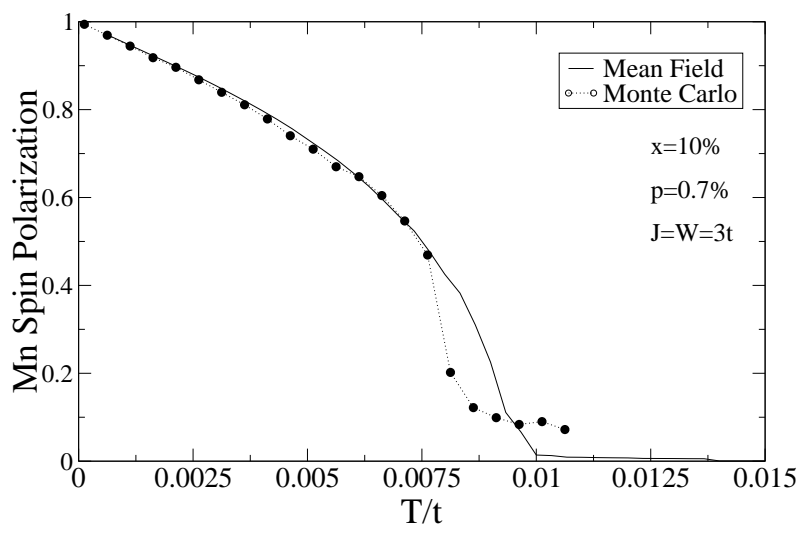

FIG. 5: Mn ions spin polarization as a function of $T$ as obtained from Monte Carlo simulations and from Weiss mean field theory. The results correspond to $x=10 \%, p=0.7 \%$, $J=W=3 t$. The MC results have been obtained in a cell of size $N=14$.

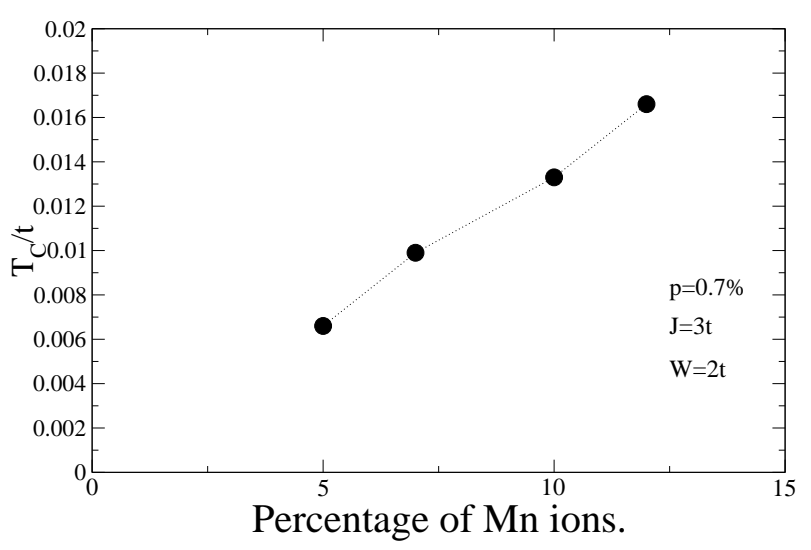

FIG. 6: Curie temperature as a function of the Mn ion's concentration as obtained from Monte Carlo simulations. The results correspond to $p=0.7 \%, J=3 t$ and $W=2 t$. The MC simulations have been done in a cell of size $N=14$.

$W=2 t$, and $p=0.7 \%$. Within the error margin, the critical temperature increases linearly with $x$, as expected for low impurity concentration.

Finally, we have studied the dependence of $T_{c}$ on the hole charge density $p$. In Fig. 7 we plot the MC results corresponding to the case $J=3 t, x=10 \%$, and two different values of $W$. For comparison, we have also plotted the Weiss mean field result. The Weiss mean field predicts a $p^{1 / 3}$ dependence, at low hole density. The MC results could be fitted perfectly to this dependence. Again we obtain that the Weiss mean field $T_{c}$ 's are higher that the MC results only for values of $W$ near the value of the exchange coupling $J$.

Before finishing our discussion on the MC results, a comparison with previous simulations is in order. Recently, Schliemann and coworkers [48] have presented some MC results for (III,Mn) V semiconductors. They work in a model similar to ours, but with the site energy difference set to zero, $W=0$. They describe the motion of 


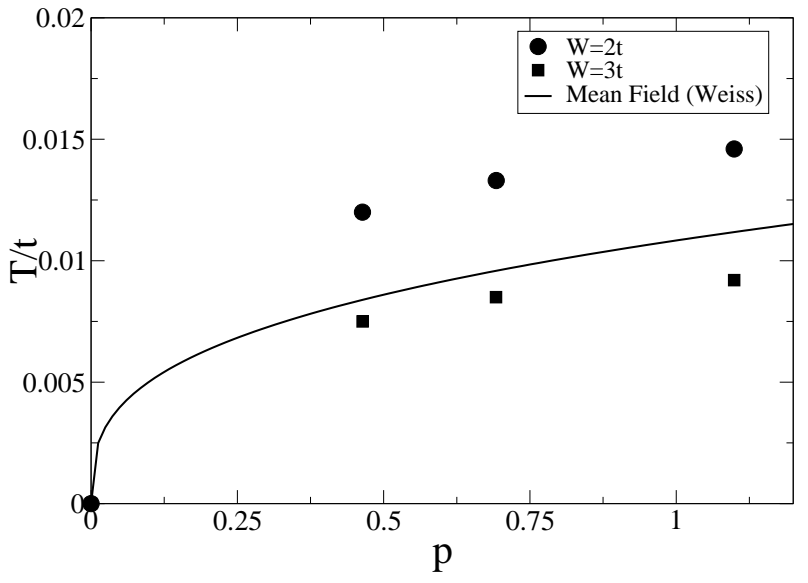

FIG. 7: Curie temperature as a function of the hole concentration, $p$, as obtained from Monte Carlo simulations and from Weiss mean field theory. The results correspond to $x=10 \%$ and $J=3 t$. The MC simulations have been done in cells of size $N=12, N=14$, and $N=16$.

the carriers using a plane wave basis with a cutoff $k_{c}$, and the exchange potential created by the magnetic impurities is modeled with a Gaussian of thickness $a_{0}$. They obtain that the critical temperature is substantially smaller than the estimated using Weiss mean field theory. This is opposite to our results: for $W=0$ our $\mathrm{MC} T_{c}$ is higher than the mean field value. As the exchange constant and the densities (Mn and carriers) used in Ref. [48] are similar to ours, there is a clear contradiction between our results and those of reference [48. A possible explanation for this discrepancy can be found in the wavelength cutoff used in ref( $[48])$, considerable larger than the impurity Gaussian width, and, perhaps, unable to trap carriers charge. If this were the case, their results could best correspond to our calculation for $W=J$, for which $T_{c}$ is smaller than $T_{c}^{V C A}$. In reference (44]), the wavelength cutoff is near $25 \AA$, whereas the value of $a_{0}$ is smaller than $5 \AA$.

A comparison with experimental results is strongly dependent on the values of the parameters. For instance, in the case of $t=0.85 \mathrm{eV}, J=3 t, x=10 \%$, and $p=0.7 \%$, the critical temperature varies between $80 \mathrm{~K}$ for $W=J$, and $350 K$ for $W=0$. This range would agree rather well with experimental data, assuming values of $W$ near $J$. Given the oversimplified nature of our model, this comparison with experiments cannot be taken too far away. In any case, our results indicate clearly the relevant role of value of $W$ on the magnetic transition: decreasing its value, if possible experimentally, would increase considerably the Curie temperature.

\section{B. CPA results.}

In this section, we present results for the phase diagram obtained with the CPA method explained in IV.

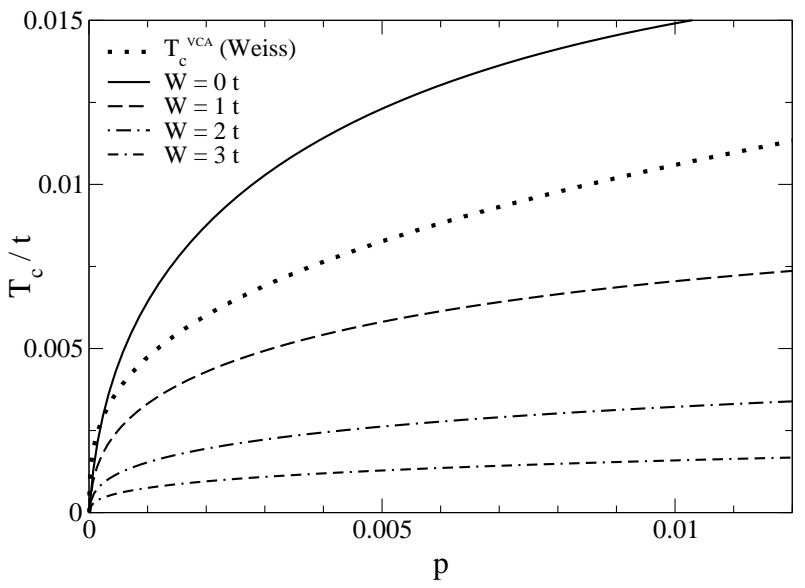

FIG. 8: CPA critical temperature as a function of hole concentration for $x=0.1, J=3 t$, and different values of $W$. The Weiss (VCA) mean field critical temperature is also plotted for comparison.

CPA results are parameterized by the density of states of the bare system, chosen to be that of the simple cubic lattice. Although unnecessary from the CPA point of view, we have assumed degenerate fermions. This is both physically correct for the relevant range of parameters, and makes the comparison with $\mathrm{MC}$ results more meaningful. We have verified that the inclusion of fermion temperature does not change results in any significant way.

In fig. 8 we present the critical temperature for $J=3 t$ as a function of carrier concentration in the relevant region, for several values of $W$. As in the MC calculations (see Fig. (1), the most important effect of $W$ is the depression of $T_{c}$. Notice, though, that the overall temperature scale of the CPA calculation is lower than that of the MC results (compare with Fig. 7). This reverses the trend found in most statistical mechanics models, where the $\mathrm{MC}$ (exact) transition temperatures are higher that the mean field counterparts. We ignore whether this effect is a genuine peculiarity of our model or rather the outcome of approximations. We observe that $T_{c}$ follows the $p^{1 / 3}$ dependence with carrier concentration expected to hold close to a 3-d band in the Weiss mean field approximation, but this agreement between $\mathrm{CPA}$ and $\mathrm{VCA}$ is restricted to the small carrier concentration region (see below). In addition, the very apparent effect of $W$ on $T_{c}$ is completely missed in the VCA, as mentioned before.

$T_{c}$ as a function of Hund coupling $J$ is presented in fig. 9, for several values of $W$. The prominent effect of $W$ in reducing the critical temperature is very clear. Comparing with the MC data of Fig. A, we see qualitatively similar curves, but, again, the MC temperatures are higher than the CPA ones. It is worth comparing the CPA results of fig. 9 with those of the VCA (Eq. 12), which predicts a dependence of the form: $T_{c}^{V C A} \propto x J^{2}$, irrespective of the size of $J$ and $x$. In the CPA treatment, this dependency only holds for small values of both 


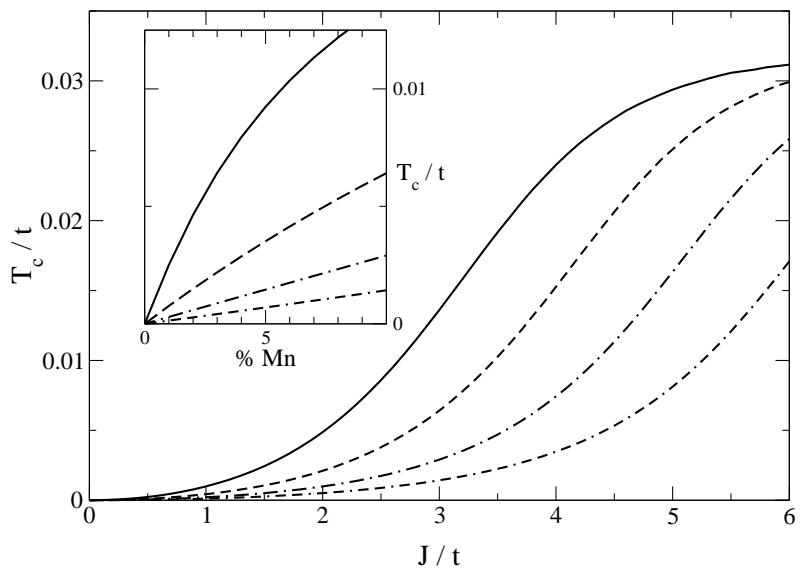

FIG. 9: CPA critical temperature as a function of $J$ for $x=0.1$ and $p=0.007$. The different curves correspond to the following values of $W$ (top to bottom): $W=0 t, 1 t, 2 t$, and 3t. Inset: Critical temperature as a function of impurity concentration for $J=3 t, p=0.007$, and the same sequence of values of $W$ (top to bottom).

$J$ and $x$, and significant deviations from the $J^{2}$ law are already evident in fig. 9, particularly for $W=0$. We have also studied the dependence of $T_{c}$ with impurity density, as shown in the inset of fig. 9 for small values of $x$. As in the MC calculations, an approximately linear regime is obtained in this limit. This linearity suggests that the effective magnetic interaction between core-spins could be well approximated by a two-body interaction in this regime. Notice that, in spite of the VCA prediction (Eq. 12), this linear dependence does not hold for higher impurity density, or even in the concentration range of fig. 9 for $W=0$, where non linear effects are already explicit. This behavior with $J$ and $x$ has been observed before for the present problem in a CPA calculation with $W=0$ and a model semicircular density of states 45 . Its survival upon inclusion of disorder $W \neq 0$ and for the real density of states (sc lattice) confirms that it is a robust feature of the model.

It has been argued earlier that the effect of $W$ on $T_{c}$ can be correlated with changes in carrier density on the impurity sites. Being the magnetic coupling mediated by mobile carriers, decreasing their density on the impurity sites (with the repulsive term $W$ ) should lead to a decrease in $T_{c}$. We can test this behavior analyzing the limit of an almost filled band, with electrons as carriers (remember that our bare fermions are holes). The effect of $W$ would then be the opposite and, therefore, $T_{c}$ should increase. To verify this idea while gaining a better perspective of the problem, we have plotted in fig. 10 the phase diagram in the whole range of carrier concentration, for three values of $W$. For $W=0$, the problem $\left(T_{c}\right)$ is particle-hole symmetric, and the ferromagnetic instability is restricted to the band edges. Although quantitatively far from the small $J$ limit, this confinement to band edges is qualitatively similar to the RKKY regime, where the same behavior can be traced back to the oscil-

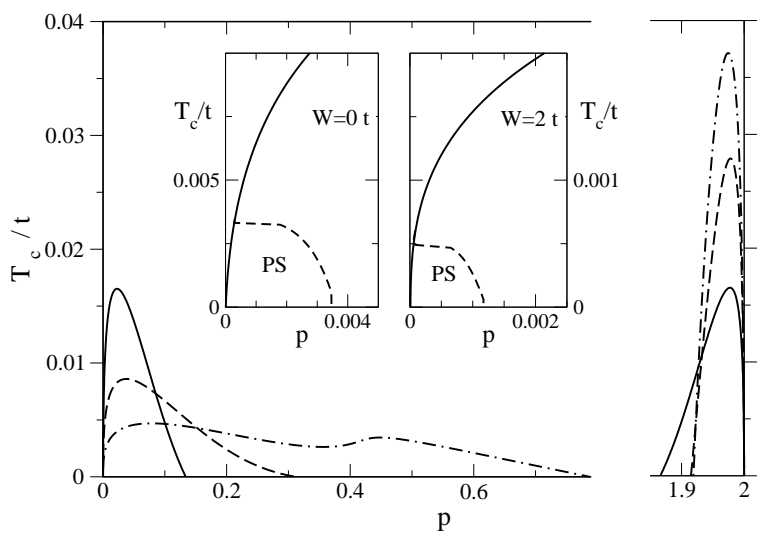

FIG. 10: CPA critical temperature as a function of hole density for $J=3 t$ and the following values of $W: W=0 t$ (continuous line), $W=1 t$ (dashed line), and $W=2 t$ (dasheddotted line). Insets: Enlarged view of the phase diagrams showing the regions of phase separation for $J=3 t$, with $W=$ $0 t$ (left inset) and $W=2 t$ (right inset).

latory nature of the magnetic coupling. As anticipated, fig. 10 confirms the asymmetric effect of $W$ on both types of carriers: it decreases $T_{c}$ at the lower edge (holes) and increases $T_{c}$ at the upper edge (electrons). Notice that, for electrons, $W$ acts as an attractive impurity, increasing the carrier density in the impurity sites, therefore increasing $T_{c}$. It is worth mentioning that fig. 10 also contains the CPA results for $W<0$ : hole density $p$ and $W>0$ is equivalent to having chosen $W<0$ and hole density $2-p$ (i.e. electron density $p$ ). From this point of view, the physics close to the upper end of fig. 10 upon increasing the value of $W$ is controlled by the development of a magnetic impurity band, with magnetic behavior becoming closer to that of the Double-Exchange model 45. Another interesting effect of increasing $W$ shows up in fig. 10: the spread of the magnetic instability from the lower edge into the band. We cannot offer a simple explanation for this fact.

Finally, we have investigated the phase diagram looking for the presence of phase separation (PS). PS is a common feature in magnetic models with itinerant carriers 49, 50, 51, 52, 53]. It seems to appear close to the magnetic transition temperature, when $T_{c}$ changes abruptly with carrier concentration 25]. In order to search for PS, we explore the existence of global minima in the thermodynamic potential, in addition to the usual local minimum employed to obtain the curve of $T_{c}$. We observe small regions, close to the band edges, where the systems is unstable versus PS. This is shown in the insets of fig. 10 for two values of $W$. Comparing results for $W=0$ and $W=2 t$, we see that the PS region decreases with increasing $W$, due to the associated reduction in the dependence of $T_{c}$ with carrier concentration. In the limit of small electron concentration, similar PS areas (not shown here) are found with opposite behavior with $W$.

As a final remark, we note that our calculation be- 
comes qualitatively similar to a recent CPA analysis [45, carried out in the absence of impurity potential: $W=0$. Quantitatively, however, it is different (even for $W=0$ ) because of our using of the real density of states (sc lattice) as opposed to a model semicircular one. Nevertheless, close to the band edges, the differences between both calculations for $W=0$ amount to a different choice of lattice regularization, or, equivalently, a rescaling of the Hund coupling $J$.

\section{SUMMARY.}

We have studied a simple model for diluted magnetic semiconductors. In this model the carriers motion is described by a tight binding Hamiltonian and the hole-Mn ion coupling has two contributions: an antiferromagnetic exchange interaction between the Mn spin and the carrier spin, $J$, and an electrostatic interaction, $W$, between the carriers charge and the potential arising from the Mn dopants. We have studied this model performing Monte Carlo simulations and mean field CPA calculations. Our main results are the following:

1) The hole charge on the Mn ions has a relevant effect on the Curie temperature. The effective impurity potential $J-W$ controls the hybridization of the $\mathrm{Mn}$ impurities with the host bands and the hole charge on the impurities. By increasing $J-W$, if possible experimentally, the charge on the $\mathrm{Mn}$ ions and the critical temperature would increase considerably.

2) The Monte Carlo critical temperature is always higher than the corresponding CPA temperature. We also find that the Monte Carlo critical temperature is in general higher than the obtained from the simplest Weiss mean field theory. Only for values of $J-W$ near cero the Weiss $T_{c}$ is higher than the Monte Carlo one.

3) The CPA calculation shows the existence of small pockets of phase segregation instability close to band edges.

\section{ACKNOWLEDGMENTS.}

We thank Prof. F. Guinea and Dr. J. Fernández Rossier for discussions. Finantial support is acknowledged from Grants No PB96-0085 (MEC, Spain) and CAM-07N/0008/2001 and CAM-07N/0015/2001 (Madrid, Spain)
[1] F. Matsukura, H. Ohno, A. Shen, and Y. Sugawara, Phys. Rev. B 57, R2037 (1998).

[2] H. Ohno, Science 281, 951 (1998).

[3] S. Sonoda, S. Shimizu, T. Sasaki, Y.Yamamoto, and H.Hori, cond-mat/0108159.

[4] S. Koshihara, A. Oiwa, M. Hirasawa, S. Katsumoto, Y. Iye, C. Urano, H. Takagi, and H. Munekata, Phys. Rev. Lett. 78, 4617 (1997).

[5] L. L. da Silva, M. A. Boselli, I. C. da Cunha Lima, X. F. Wang, and A. Ghazali, Appl. Phys. Lett. 79, 3305 (2001).

[6] J. Fernández-Rossier and L. J. Sham, Phys. Rev. B 64, 235323 (2001).

[7] L. Brey and F. Guinea, Phys. Rev. Lett. 85, 2384 (2000).

[8] T. Jungwirth, W. A. Atkinson, B. H. Lee, and A. H. MacDonald, Phys. Rev. B 59, 9818 (1999).

[9] J. Szczytko, A. Twardowski, K. Swiatek, M. Palczewska, M. Tanaka, T. Hayashi, and K. Ando, Phys. Rev. B 60, 8304 (1999).

[10] M. Linnarsson, E. Janzen, B. Monemar, M. Kleverman, and A. Thilderkvist, Phys. Rev. B 55, 6983 (1997).

[11] H. Ohno and F. Matsukura, Solid State Commun. 117, 179 (2001).

[12] H. Ohno, A. Shen, F. Matsukura, A. Oiwa, A. Endo, S. Katsumoto, and Y. Iye, Appl. Phys. Lett. 69, 363 (1996).

[13] A. V. Esch, L. V. Bockstal, J. D. Boeck, G. Verbanck, A. S. van Steenbergen, P. J. Wellmann, B. Grietens, R. Bogaerts, F. Herlach, and G. Borghs, Phys. Rev. B 56, 13103 (1997).

[14] T. Dietl, H. Ohno, F. Matsukura, J. Cibert, and D. Ferrand, Science 287, 1019 (2000).

[15] T. Dietl, H. Ohno, and F. Matsukura, Phys. Rev. B 63, 195205 (2001).
[16] J. Konig, H. H. Lin, and A. H. MacDonald, Phys. Rev. Lett. 84, 5628 (2000).

[17] T. Jungwirth, J. Konig, J. Sinova, J. Kucera, and A. H. MacDonald, cond-mat/0201157.

[18] M. Berciu and R. N. Bhatt, Phys. Rev. Lett. 87, 7203 (2000).

[19] R. N. Bhatt, M. Berciu, M. P. Kennet, and X. Wan, cond-mat/0111184.

[20] A. Kaminski and S. D. Sarma, cond-mat/0201229.

[21] S.-R. E. Yang and A. H. MacDonald, cond-mat/0202021.

[22] Y. Tokura, J. Magn. Magn. Mater. 200, 1 (1999).

[23] K.-I. Kobayashi, T. Kimura, H. Sawada, K. Terakura, and Y. Tokura, Nature 395, 677 (1998).

[24] Y. Shimikawa, Y. Kubo, and T. Manako, Nature 379, 53 (1996).

[25] F. Guinea, G. Gómez-Santos, and D. Arovas, Phys. Rev. B 62, 391 (2000).

[26] P. Majumdar and P. B. Littlewood, Phys. Rev. Lett. 81, 1314 (1998).

[27] M. J. Calderón, L. Brey, and P. B. Littlewood, Phys. Rev. B 62, 3368 (2000).

[28] J. B. M. Albolfath, T. Jungwirth and A. H. MacDonald, Phys. Rev. B 63, 054418 (2001).

[29] J. Konig, J. Schliemann, T. Jungwirth, and A. H. MacDonald, cond-mat/0111314.

[30] T. Omiya, F. Matsukura, T. Dietl, Y. Ohno, T. Sakon, M. Motokawa, and H. Ohno, Physica E 7, 976 (2000).

[31] J. Okabayashi, A. Kimura, O. Rader, T. Mizokawa, A. Fujimori, T. Hayashi, and M. Tanaka, Phys.Rev.B 58, R4211 (1998).

[32] S. Sanvito, P. Ordejón, and N. A. Hill, Phys. Rev. B 63, 165206 (2001).

[33] In the interaction between the ion spins and the fictitious 
field $\mathbf{B}_{f}(I)$ we take $g \mu_{B}=1$.

[34] M. J. Calderón and L. Brey, Phys. Rev. B 58, 3286 (1998).

[35] J. A. Vergés, V. Martín-Mayor, and L. Brey, Phys. Rev. Lett (2002), in press.

[36] D. W. Taylor, Phys. Rev. 156, 1017 (1967).

[37] P. Soven, Phys. Rev. 156, 809 (1967).

[38] R. J. Elliott, J. A. Krumhansl, and P. L. Leath, Rev. Mod. Phys. 46, 465 (1974).

[39] A. Georges, G. Kotliar, W. Krauth, and M. Rozenberg, Rev. Mod. Phys. 68, 13 (1996).

[40] J. Alonso, L. A. Fernández, F. Guinea, V. Laliena, and V. Martín-Mayor, Phys. Rev. B 63, 054411 (2001).

[41] E. N. Economou, Green's Functions in Quantum Physics (Springer-Verlag, Berlin, 1983).

[42] M. Auslander and E. Kogan, cond-mat/0106032.

[43] N. Furukawa, J. Phys. Soc. Jap. 64, 2734 (1995).

[44] N. Furukawa, in Physics of Manganites, edited by T. Kaplan and S. Mahanti (Kluwer/Plenum Publisher, New
York, 1999).

[45] A. Chattopadhyay, S. D. Sarma, and A. J. Millis, Phys. Rev. Lett. 87, 227202 (2001).

[46] M. Auslander and E. Kogan, cond-mat/0111491.

[47] G. Gómez-Santos, unpublished work.

[48] J. Schliemann, J. Konig, and A. H. MacDonald, Phys. Rev. B 64, 165201 (2001).

[49] E. L. Nagaev, Physica B 230-232, 816 (1997).

[50] D. P. Arovas and F. Guinea, Phys. Rev. B 58, 9150 (1998).

[51] E. Dagotto, S.Yunoki, A.L.Malvezzi, A.Moreo, J.Hu, S.Capponi, D.Poilblanc, and N.Furukawa, Phys. Rev. B 58, 6414 (1998).

[52] D. Arovas, G. Gómez-Santos, and F. Guinea, Phys. Rev. B 59, 13569 (1999).

[53] A. Moreo, S. Yunoki, and E. Dagotto, Science 283, 2034 (1999). 